\title{
CHARACTERISTICS OF DOG SEMEN AFTER DILUTION AND COOLING
}

\author{
MESSIAS,C. ${ }^{1}$; AMARAL, M.C. ${ }^{1}$; WEISS, R.R. ${ }^{2}$
}

${ }^{1}$ Médicas Veterinarias autonomas;

${ }^{2}$ Departamento de Medicina Veterinaria - UFPR.

The purpose of this study was to evaluate the efficiency of four different diluents for conservation of canine smen stored at $5^{\circ} \mathrm{C}$. For the experiment were selected two mixed breed dogs with the same age and size. Ten ejaculates were collected from each dog during five weeks. The samples were diluted in TRIS-egg yolk, skim milk, TRIS-egg yolk in addition to prostatic fluid and skim milk in addition to prostatic fluid. The samples were evaluated before dilution; immediately after (time 0); 24, 48, 72 and 96 hours after cooling. Samples of undiluted semen were evaluated in the same way for comparison purposes. The spermatological evaluation concerned progressive motility and acrosome integrity after dilution. The results were: 1 . TRIS-egg yolk was superior to skim milk in regard to progressive motility and as efficient as skim milk in regard to acrosome integrity. 2. The diluents TRIS-egg yolk and skim milk, when added to prostatic secretion, were always inferior in regard to progressive motility and acrosome integrity. 3 . Time of the semen viability with at least $50 \%$ of progressive motility was as follows: for TRIS-egg yolk, 82,5 hours ; for skim milk, 60,7 hours; for TRIS-egg yolk+prostatic fluid, 60,3 hours; for skim milk+prostatic fluid, 38,4 hours. 4 . The addition of diluents addicted to prostatic fluid did not show satisfactory results on the dog semen conservation at $5^{\circ} \mathrm{C}$.

Key Words: dog, semen, cooled semen. 